\title{
Optimized staircase profiles for diffractive optical devices made from absorbing materials
}

\author{
Bernd Nöhammer, Christian David, and Jens Gobrecht \\ Laboratory for Micro- and Nanotechnology, Paul Scherrer Institut, CH-5232 Villigen, Switzerland \\ Hans Peter Herzig \\ Institute of Microtechnology, University of Neuchâtel, CH-2000 Neuchâtel, Switzerland
}

Received March 11, 2003

\begin{abstract}
We report on the optimization of staircase grating profiles for the case of absorbing grating materials. Using a simple numerical algorithm, we determined the grating parameters, maximizing the first-order diffraction efficiency for different numbers of staircase steps. The results show that there is a significant difference between the staircase profiles for nonnegligible and negligible absorption. The obtained solutions are of importance for diffractive optics in the soft-x-ray and extreme-ultraviolet ranges. () 2003 Optical Society of America

OCIS codes: $\quad 050.1960,050.1970,220.3740,260.7200,340.0340$.
\end{abstract}

Because of the progress in lithography and replication techniques that permit low-cost mass fabrication, diffractive optical elements (DOEs) have become important optical devices. ${ }^{1}$ The most important and also simplest form of DOE is gratings. There are also many other types of DOE that are generalized forms of gratings with a varying grating constant, such as Fresnel zone plates or computer-generated holograms. Consequently the task of finding the optimum surface relief of a DOE can often be simplified to the problem of finding the optimum shape of a grating.

In conventional optics the absorption of the grating material is usually negligible; therefore only the phaseshifting properties of the material (described by the real part of the refractive index) have to be taken into account for this shape-optimization process. However, for wavelengths $\lambda$ in the extreme-ultraviolet (EUV) and $\mathrm{x}$-ray ranges, where the refractive index $n$ is conveniently written as $n(\lambda)=1-\delta(\lambda)+i \beta(\lambda), \delta$ and $\beta$ are often of the same order of magnitude; therefore absorption (described by $\beta$ ) also has to be taken into account for the calculation of optimized grating profiles.

A requirement for many applications is to diffract as much light as possible in a single (e.g., the first) diffraction order. In the case in which absorption is negligible, this is achieved by use of a blazed grating structure that has a sawtoothlike shape and a height $h_{c}$ calculated from $h_{c}=\lambda /|\delta|$ [Fig. 1(A), dashed lines]. However, when absorption of the diffracting structures plays a role, the shape of the grating that gives maximum first-order diffraction efficiency is quite different. Tatchyn et $a l .{ }^{2}$ have shown that in this general case the optimum profile is still sawtooth shaped and has the same slope as in the case of zero absorption [Fig. 1(A), solid lines], but the structures are narrower, resulting in an open part $b_{1}$. The size of the open fraction $b_{1} / b$ depends on the ratio $\delta / \beta$ as indicated in Fig. 1(B) and has to be calculated numerically.

In practice, fabrication of a continuous sawtooth profile with the required accuracy is difficult. There- fore the ideal profile is often approximated by a staircase profile (Fig. 2). Such staircase profiles can be fabricated by use of several aligned lithography steps and subsequent etching or deposition of the grating material. ${ }^{3}$

For the case of staircase profiles optimized for negligible absorption, the $N$ distinct steps of the staircase have equal width $\left(w_{i}=w_{j} ; i, j=1, \ldots, N\right)$ and the heights, $h_{i}$, of the steps follow the ideal profile for zero absorption, leading to $h_{i}=[(i-1) / N] \lambda /|\delta|$. Such profiles give good results for optics in the visible spectral range, where absorption is normally negligible. Also, in the case of the first x-ray optics with staircase profiles, which have been recently reported, ${ }^{4-6} \beta$ was sufficiently small for the relevant photon energies in the hard-x-ray range. However, when considering optics in the EUV and soft-x-ray region, in most cases absorption will play a role. In the current work we show how to optimize the design of a staircase profile to obtain maximum first-order diffraction efficiency for this case of nonnegligible absorption.

In the EUV and soft-x-ray spectral ranges, the wavelength of the light is typically small compared
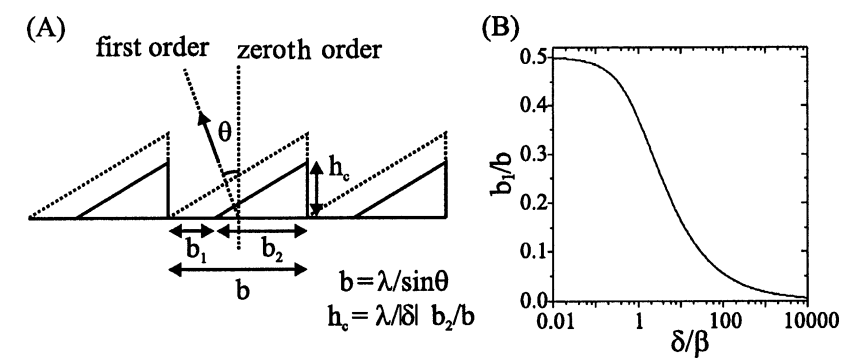

Fig. 1. (A) Design of a transmission grating maximizing the first-order diffraction efficiency in the case of absorbing grating material (solid lines) and in the case of negligible absorption (dashed lines). (B) Correlation between free design parameter $b_{1}$ and the optical properties of the grating material (quantified by $\delta / \beta$ ). 


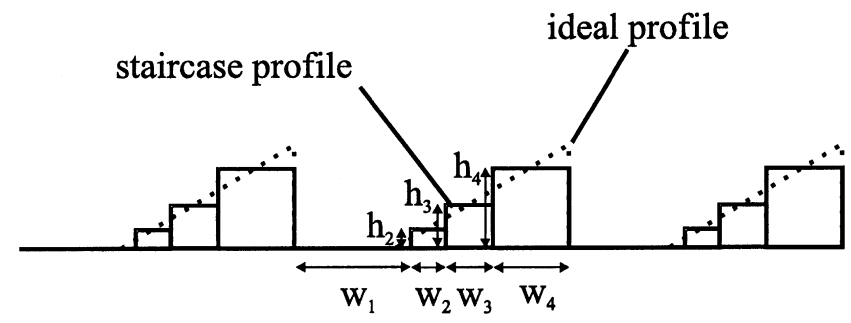

Fig. 2. General form of a staircase profile enabling the optimization of the first-order diffraction efficiency.

with the period $b$ and the height of the gratings used; therefore in most cases a grating can be treated as a thin structure, and the thin-element approximation can be used (for a discussion of the validity of the thinelement approximation see, for example, Ref. 1). In addition, $\delta$ and $\beta$ are typically very small; consequently reflections at surfaces between different materials are negligible. With these approximations the diffraction efficiency $\eta$ can be calculated with the aid of a Fourier analysis of the transmission function. For a discrete profile we get $^{1}$

$$
\eta=\left|\sum_{i=1}^{N} \phi_{i}\right|^{2}
$$

where

$$
\phi_{i}=\exp \left(-2 \pi \underline{h}_{i} \beta / \delta\right) \exp \left[2 \pi i\left(\underline{h}_{i}-\underline{x}_{i}\right)\right] \sin \left(\pi \underline{w}_{i}\right) / \pi,
$$

and the normalized heights $\left(\underline{h}_{i}\right)$, widths $\left(\underline{w}_{i}\right)$ and middle positions $\left(\underline{x}_{i}\right)$ of the steps are denoted

$$
\underline{h}_{i}=h_{i}|\delta| / \lambda, \quad \underline{w}_{i}=w_{i} / b, \quad \underline{x}_{i}=x_{i} / b .
$$

Equation (1) is a sum of contributions from each step of the staircase profile shown in Fig. 2. The first term in Eq. (2) describes the absorption within one step. The second term gives the phase of each contribution, originating from the material phase shift $\left(h_{i}\right)$ and the position of the step within the grating $\left(x_{i}\right)$. The third term is obtained from the Fourier transform of a rectangular function with width $w_{i}$. The analytical treatment of the problem would be rather complex since the efficiency is a complicated sum over functions of all staircase parameters. Therefore we used a numerical approach, applying a local search algorithm ${ }^{7}$ to find the optimum values for the parameters in Eq. (3) with respect to diffraction efficiency.

The principle of the algorithm is to make small, random, trial changes in the actual profile, where the change is allowed to take place only if the new profile (the new set of parameters $h_{i}, w_{i}$, and $x_{i}$ ) has a higher diffraction efficiency than the previous one. By repeating this step until a large number $\left(n_{\text {trial }}>100\right)$ of subsequent trial changes fails to improve the diffraction efficiency, an optimum set of values of $h_{i}, w_{i}$, and $x_{i}$ will ultimately be reached.

This kind of algorithm could fail because of the presence of local maxima and as a consequence would never yield reasonable results for the design parameters of the grating. However, the numerical results show that the total number of maxima is rather small; therefore it is sufficient to repeat the whole algorithm a few (typically 20) times with different, randomly chosen starting parameters for the grating in order to obtain a parameter set that represents the global optimum with respect to diffraction efficiency.

Figure 3 shows the numerical results of using this algorithm in the case of a staircase profile with four levels. For low values of absorption (high values of $\delta / \beta)$ we obtain the expected staircase profile, which has steps with equal width and normalized heights of $0,1 / 4,1 / 2$, and $3 / 4$. When we go to higher values of absorption, the widths (as well as the heights) of the second and the third steps decrease and finally approach zero. The widths of the first and the fourth steps both increase, and for values of $\delta / \beta$ near zero they both approach a value equal to half of the grating period $b$. Therefore in the limit of infinite absorption a conventional binary amplitude grating is obtained. These results for high values of absorption can be qualitatively understood because the staircase profile always has to provide an optimum approximation of the ideal profile in terms of diffraction efficiency. For the ideal grating in the case of strong absorption the main contributions to the diffraction efficiency come from regions of the grating with small height.

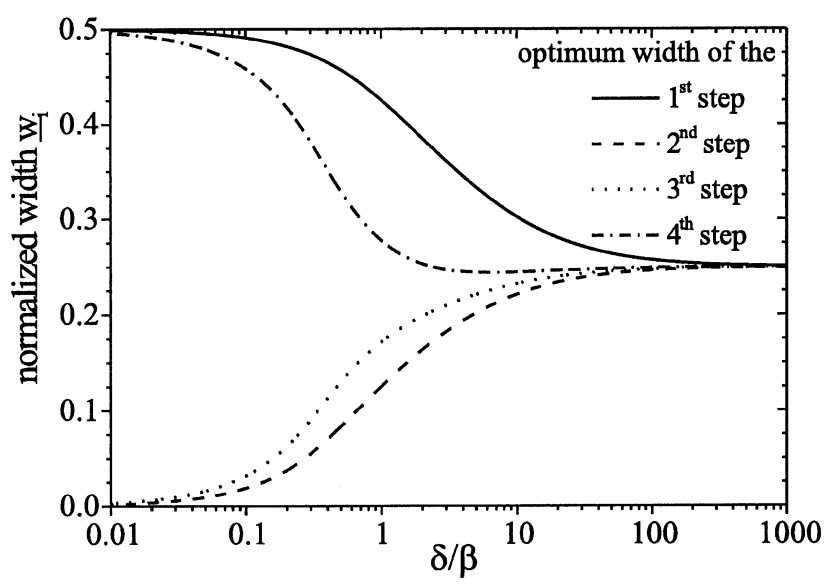

(A)

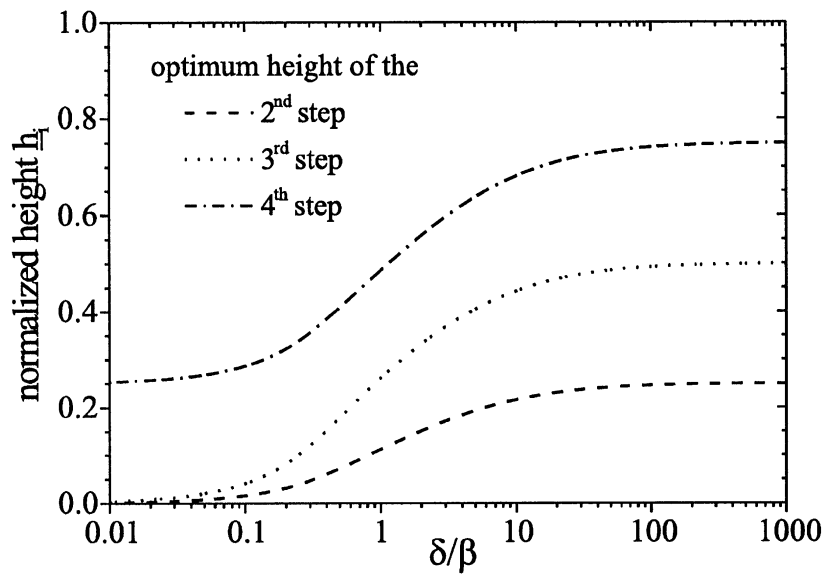

(B)

Fig. 3. Optimum normalized widths $\underline{w}_{i}$ (A) and heights $\underline{h}_{i}$ (B) of a four-step staircase profile as a function of $\delta / \beta$ of the grating material. 


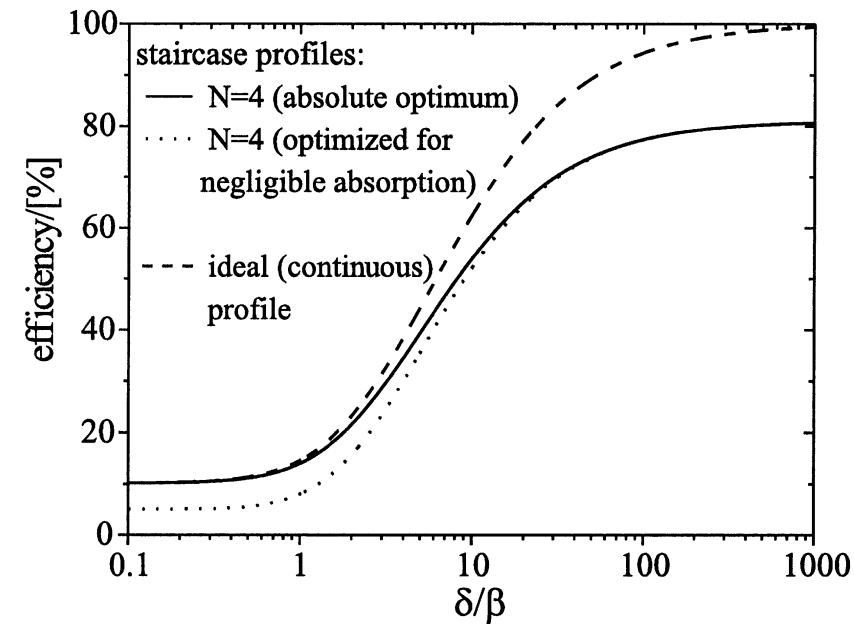

Fig. 4. First-order diffraction efficiency of a four-step profile (solid curve) featuring optimal values for the heights and widths of the steps. In addition, the diffraction efficiency of the ideal continuous profile (see Fig. 1) and a four-step profile optimized for negligible absorption are depicted.

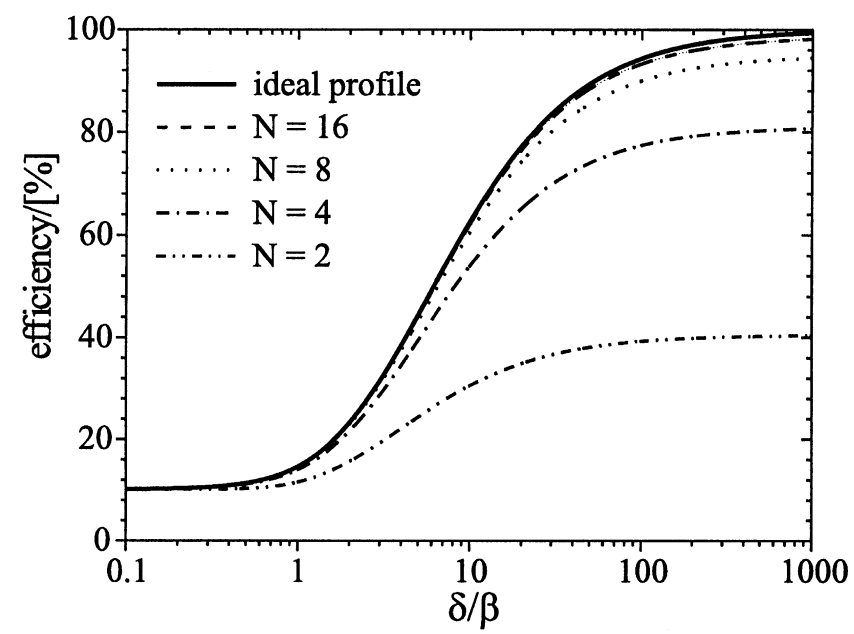

Fig. 5. First-order diffraction efficiency for different optimized grating designs.

Therefore it is important to get a good approximation of the region with zero height (provided by the first step with $w_{1} \approx b_{1}$ ) and a short region with small height at the beginning of the sawtooth (provided by the second and third steps, which therefore tend to have rather small widths and heights in the case of high absorption).

In Fig. 4 the diffraction efficiency of a four-step profile with optimal values for $h_{i}, w_{i}$, and $x_{i}$ is depicted. In addition, the diffraction efficiency of the ideal continuous profile and a four-step profile with the conventional design rule optimized for zero absorption are plotted for comparison. For high values of $\delta / \beta$ the two different staircase profiles nearly have the same diffraction efficiency, whereas for values of $\delta / \beta$ below 10 a significant difference can be observed. Therefore the optimal design of the staircase profile has to be used in this case to guarantee maximum diffraction efficiency (e.g., for $\delta=2$ the optimum four-step profile gives $22 \%$ diffraction efficiency in comparison with only $16 \%$ for a four-step profile optimized for zero absorption).

When different step numbers $N$ of the staircase profile are used, similar results are found. For high values of $\delta / \beta$ (typically $\delta / \beta>10$ ) the anticipated profile optimized for zero absorption $\left[w_{i}=w_{j}\right.$ and $\left.h_{i}=(i-1) / N\right]$ is obtained. For the case of high absorption the staircase profile gives a good approximation of the ideal continuous profile in regions with small or zero structure height and an inferior approximation in regions of large structure heights, as expected. Figure 5 shows the first-order diffraction efficiencies of optimized profiles with different step numbers $N$. For small values of absorption a larger number $N$ of steps leads to a strongly improved diffraction efficiency, whereas for strong absorption very little difference is found among all profiles. This is because, for all profiles, the normalized width of the region with zero height approaches $1 / 2$, going to small values of $\delta / \beta$. Consequently in the limit of infinite absorption all the staircase profiles will have the same optical properties as a binary amplitude grating.

In summary, we have shown that material absorption has to be taken into account for the optimization of gratings in the EUV and x-ray ranges. By use of a numerical local search algorithm we were able to calculate the optimum parameters for maximum firstorder diffraction efficiency of gratings with staircase profiles.

This work was funded by the Swiss National Science Foundation. B. Nöhammer's e-mail address is bernd.noehammer@psi.ch.

\section{References}

1. H. P. Herzig, ed., Micro-optics (Taylor \& Francis, London, 1998).

2. R. Tatchyn, P. L. Csonka, and I. Lindau, J. Opt. Soc. Am. 72, 1630-1639 (1982).

3. M. B. Stern, in Micro-optics, H. P. Herzig, ed. (Taylor \& Francis, London, 1998), pp. 53-86.

4. E. Di Fabrizio, F. Romanato, M. Gentili, S. Cabrini, B. Kaulich, J. Susini, and R. Barrett, Nature 401, 895-898 (1999).

5. W. Yun, B. Lai, A. A. Krasnoperova, E. Di Fabrizio, Z. Cai, F. Cerrina, Z. Chen, M. Gentili, and E. Gluskin, Rev. Sci. Instrum. 70, 3537-3541 (1999).

6. B. Nöhammer, J. Hoszowska, H. P. Herzig, and C. David, presented at the X-Ray Microscopy Conference 2002, Grenoble, France, July 29-Aug ust 2, 2002.

7. E. Aarts and J. K. Lenstra, Local Search in Combinatorial Optimization (Wiley, Chichester, U.K., 1997). 